Resumen: El objetivo de este escrito es reflexionar sobre las características del pensamiento proyectual, propio de las disciplinas de Diseño. Este tipo de pensamiento, por romper con otras modalidades y tradiciones cognitivas, se presenta hoy en día como una oportunidad para diversas disciplinas.

Palabras claves: Pensamiento de diseño - Pensamiento proyectual - investigación en diseño - prospectiva

[Resúmenes en inglés y portugués en la página 72]

${ }^{(*)}$ Arquitecta, UM. Profesora Superior Universitaria, UM. Magister en gestión de proyectos educativos, CAECE. Doctora en Arquitectura, FADU-UBA. Docente e Investigadora de las Universidades de Buenos Aires y Palermo.

\title{
Aproximaciones para una Epistemología del Diseño
}

Si la Filosofía de la ciencia se ocupa de pensar la ciencia, constituyendo una reflexión de segundo orden, es decir un pensamiento sobre el pensamiento, en este caso el científico; podríamos, de modo análogo, crear una filosofía o epistemología del diseño, cuyo objeto de estudio sería, entonces, el pensamiento de diseño.

A los fines de abordar esta Epistemología de Diseño será condición sine qua non definir en qué consiste el pensamiento de diseño.

Vale señalar que este concepto en las últimas décadas tomó significativa proyección, trasladándose del campo específico de las disciplinas proyectuales al área de los negocios y la gestión.

En su origen la noción de “pensamiento de diseño" hacía referencia a las estrategias creativas que habitualmente son empleadas por los diseñadores durante el proceso de diseño. Y es por eso que esta noción estuvo inicialmente imbricada con las "metodologías de diseño”. En este sentido, Bruce Archer (1965), ya a comienzos de la década del sesenta, sostiene que el diseño "no es solo una habilidad artesanal, sino que debe considerarse una disciplina basada en el conocimiento por derecho propio, con metodología rigurosa y princi- 
pios de investigación incorporados en el proceso de diseño". En su libro "Método sistemático para diseñadores" el mismo Archer hará hincapié en enumerar todo los tipos de saberes que son necesarios para concretar el "pensamiento de diseño".

En esos mismos años, desde un enfoque más positivista, Herbert Simon, propone concebir al diseño como una ciencia, planteando la necesidad de estudiar científicamente el mundo creado por el hombre y mirar al diseño como una ciencia de lo artificial en donde sería de crucial importancia la toma de decisiones.

Mario Bunge (1989) suma mayor claridad cuando señala que "los conceptos clave de la técnica y de la tecnología son los de objeto artificial (a diferencia del natural) y de diseño del mismo (a diferencia de una representación a posteriori). Todo objeto artificial ha sido diseñado y todo diseño lo es de un artefacto (posible o imposible)"

Para Simon (1996, p. 113), entonces, la ciencia de diseño sería "una doctrina intelectualmente dura, analítica, parcialmente formalizable, parcialmente empírica, enseñable sobre el proceso de diseño".

Tiempo más tarde Archer (1979, p. 17) precisa este concepto señalando que "Existe una forma de pensamiento y comunicación que es a la vez diferente de las formas científicas $y$ académicas de pensamiento y comunicación, y tan poderosa como los métodos científicos y académicos de investigación cuando se aplica a sus propios tipos de problemas".

En 1982 Nigel Cross (2001) publicó un artículo iniciático "Designerly Ways of Knowing', que daría lugar a una larga serie de reflexiones, donde señalaba que el diseño poseía una cultura propia, intelectual y práctica, como base para la enseñanza, diferente de la de las Ciencias Naturales, las Artes y las Humanidades. Esto lo lleva a afirmar que "Hay cosas que conocer, formas de conocerlas y maneras de averiguar sobre ellas que son especificas del área de diseño." Cross sostiene que la enseñanza ha estado habitualmente orientada a responder a dos tipos de cultura: La de las ciencias naturales y la de las humanidades. Mientras que la primera plantea como valor la objetividad, la racionalidad, la neutralidad y la búsqueda de la verdad; la segunda se enfoca a la subjetividad, la reflexión, la comprensión y la necesidad de realizar interpretaciones. El diseño como cultura posee valores diferentes a estas dos tradiciones. Sabemos además que las ciencias naturales utilizan una metodología basada en la observación, el experimento y el análisis, mientras que las humanidades se apoyan en la interpretación, la analogía y la metáfora. El diseño, a diferencia de otras disciplinas, se sustenta en la creación de modelos, la formulación de patrones y la síntesis. Similar aseveración realiza Roberto Doberti desde "La Cuarta Posición".

Sin embargo, hay otra cuestión que diferencia a las ciencias naturales y humanas de las disciplinas "de lo artificial", esto es que tanto el diseño y la tecnología no sólo producen conocimiento, sino que además producen artefactos.

Aclaremos un poco más esta afirmación: Un problema científico responde a la pregunta: “Por qué?”, mientras que los problemas tecnológicos y de diseño responden a un interrogante del tipo de “¿Cómo?” o ¿De qué manera...?” Esto se debe a que la ciencia tiene como objeto la producción de conocimiento, mientras que la tecnología y el diseño producen no sólo un saber proposicional y verbalizable, sino también artefactos, entendidos éstos de la manera más amplia: objetos, instrumentos, procesos, planes, hasta organismos vivos, es decir objetos artificiales creados por el hombre. Es decir, el diseño genera un do- 
ble producto: El artefacto y el conocimiento. Los criterios de evaluación del conocimiento y el artefacto son disímiles: El conocimiento se evalúa en términos de verdadero/falso, mientras que el artefacto implica considerar su eficacia, su eficiencia y la satisfacción de ciertos valores. En la tecnología se puede hablar de valores de cuidado ambiental y social, mientras que en el diseño estos valores pueden ser estéticos, estilísticos, sustentables, culturales, simbólicos, etc.

El énfasis que realiza el diseño en torno a la producción del artefacto hace olvidar a veces el conocimiento obtenido.

En 1987 Peter Rowe (1991) en su libro Design Thinking da cuenta sistemáticamente de cómo son los procedimientos de resolución de problemas que emplean los arquitectos y urbanistas, considerando entonces al diseño como una actividad intelectual en la que se toma decisiones sobre la base de ciertos principios guía.

Es por ello que el pensamiento de diseño se focaliza primero en la definición del problema, para luego encarar su solución. Este punto también es clave en el campo de diseño y la tecnología, puesto que los principiantes no se entrenan en la resolución de problemas ya planteados, sino que deben definir en qué consiste el problema a fin de poder presentar una posible solución del mismo. En sí misma, esta redefinición del problema va a ampliar el tipo de propuestas resolutivas que se propongan, en función de los aspectos que se hayan considerado relevantes en el problema. Es por ello que, si en las disciplinas científicas un problema tiene una solución correcta (o unas pocas), en el campo del diseño y de la tecnología una situación da a lugar a múltiples problemas, cada uno de los cuales tiene, a su vez, múltiples soluciones correctas, que no pueden ser calificadas en términos exhaustivos de verdaderas o falsas sino como un gradiente continuo que va desde las mejores a las peores soluciones.

Recordemos que Donald Schon (1998) decía. "Un diseñador juega con variables, reconcilia los valores en conflicto y transforma los impedimentos. Se trata de un proceso en el que, aún a sabiendas de que unos productos diseñados pueden ser mejores que otros, no existe una única respuesta válida...."

Bruce Archer (1992) diferencia en las ciencias de lo artificial, entre el diseño y la tecnología considerando que "Si la tecnología es "saber cómo", entonces el diseño es «imaginar qué». La capacidad de prever una realidad no presente, analizándola y modelándola externamente, es la tercera gran definición característica de la humanidad, junto con la fabricación de herramientas y el uso del lenguaje".

Esta capacidad de anticipar, de imaginar, de "proyectarse" es clave en las disciplinas de diseño. En este sentido Roberto Fernández (2013, p. 40) sostiene que "En relación con la tendencia a identificar proyectos con dispositivos empiricos de prefiguración de futuros, también decimos que la investigación proyectual puede estar caracterizada por dicha práctica; es decir, se pueden producir proyectos de futuros no como actividades extremadamente arbitrarias o subjetivas sino como parte de estrategias cognitivas, es decir, como dispositivos típicos de investigación ligados a la función del descubrimiento, la anticipación o la prognosis..." 


\section{Anticipaciones}

Es necesario señalar que para poder avanzar en nuestra argumentación se debe tener en cuenta que tanto la tecnología como el diseño tienen una orientación al futuro.

Sin embargo, en el pensamiento humano encontramos distinto tipo de anticipaciones conceptuales. La respuesta a este tipo de interrogante asume caminos distintos que es necesario recorrer.

\section{Prediccciones}

En primer lugar tenemos a las "Predicciones científicas", las cuales se nos presentan como argumentos de forma condicional (si... entonces...) en el que el suceso anticipado surge como consecuencia lógica de la aparición de ciertas condiciones iniciales y de alguna ley ${ }^{1}$ científica. (Klimovsky-Hidalgo, 1998) (Gaeta, 1996) (Schuster, 2005)

Es el modelo de anticipación de futuro que proponen las ciencias naturales para los sistemas estables. Ej.

Si se dan las condiciones A, entonces se producirá el evento B (Ley)

Suceden las condiciones A (Condiciones iniciales)

Por lo tanto se dará el fenómeno B

La ley puede ser probabilística de modo que:

Si se dan las condiciones A, entonces se producirá el evento B con cierta probabilidad (Ley)

Suceden las condiciones A (Condiciones iniciales)

Por lo tanto es probable que se cumpla el fenómeno B

Existen dificultades para poder realizar predicciones certeras:

Algunos campos de la ciencia presentan dificultades para realizar predicciones exactos. Esto puede deberse a diferentes causas:

Variables ocultas no conocidas. Muchas veces en un proceso natural (o cultural) intervienen junto con factores relevantes bien identificados, medibles y cuantificables, otros factores cuya presencia o ausencia es difícil de determinar, y cuya aparición, a veces, tiene una influencia determinante en el resultado final de proceso. Estos factores cuya presencia es difícil o imposible de determinar es lo que se denomina "variable oculta".

Señala Ilya Prigogine (1996) “...tanto en Dinámica Clásica como en Física Cuántica las leyes fundamentales ahora expresan posibilidades, no certidumbres. No sólo poseemos leyes, sino acontecimientos que no son deducibles de las leyes pero actualizan sus posibilidades", es por ello que considera que "La formulación de las leyes físicas debe ser modificada en todos los niveles con el fin de estar de acuerdo con el universo abierto y evolutivo en que viven los humanos".

Ej. Imposibilidad de predecir terremotos con exactitud

Ej. Imposibilidad de predecir cambios sociales con precisión. 
Dinámica desconocida o compleja. No todos los sistemas son estables puesto que sucede a veces que aún conociendo las variables relevantes para predecir el resultado de un proceso, las relaciones entre estas variables no se conoce con precisión, o implican intrincados modelos matemáticos multivariables dependientes de la medición rigurosa de complejas condiciones iniciales que determinan resultados probablísticos.

Esto a veces se lo conoce como "efecto mariposa", ya que pequeños cambios en las condiciones iniciales pueden dar lugar a enormes discrepancias en los resultados.

Ej. La predicción del tiempo meteorológico sólo es posible con unos pocos días de antelación. Ej. Predicciones en genética.

Fenómenos aleatorios: Existen fenómenos cuyo comportamiento es aleatorio, lo cual implica la imposibilidad de formular leyes determinísticas ${ }^{2}$. Un ejemplo es principio de indeterminación o de incertidumbre Heisenberg que establece la imposibilidad de que determinados pares de magnitudes físicas sean conocidas con precisión arbitraria.

\section{Pronósticos}

El segundo tipo de anticipación es el que se denomina "Pronóstico", siendo éste una afirmación condicional en la que se anticipa la ocurrencia de un hecho sobre la base de la presencia de ciertas condiciones antecedentes. Se fundan en tendencias ${ }^{3}$, o generalizaciones empíricas que no tienen el carácter de leyes, puesto que no existen en las disciplinas que se emplean, leyes causales o determinísticas. Esto se da frecuentemente en economía y demografía que son ciencias sociales. (Marques, 1999)

Vale aclarar que las generalizaciones acerca del comportamiento humano no poseen las características que presentan las leyes de las ciencias naturales dado que:

Las variables que intervienen (creencias/deseos/conducta) no pueden medirse de manera independiente y se encuentran condicionadas espacio-temporalmente y por diversas condiciones sociales y culturales. (Nagel, 2006)

Requieren la aceptación de un supuesto básico indemostrable o vacío: la racionalidad de toda conducta humana. (Hempel, 1979) (Salmon, 2002)

Implican la imposibilidad de reducir los conceptos intencionales a estímulos físicos, medibles de manera más objetiva.

\section{Profecías}

El tercer tipo de anticipación es el que se conoce como "Profecía", es decir, una afirmación incondicional ("sucederá A") que supone la ocurrencia de una hecho futuro sin presentar fundamentos empíricos que lo hagan esperable. Carece de valor científico y es un hecho fantasioso y meramente especulativo. De acuerdo con el diccionario de la Real Academia es " $u n$ don sobrenatural que consiste en conocer por inspiración divina las cosas distantes of futuras". Las profecías están ligadas a prácticas religiosas, seudo científicas -como la astrología- , y a referencias apocalípticas -como el fin del mundo que iba a ocurrir en el 2012-. Muchas veces son enunciadas con un alto grado de imprecisión que las hacen, de acuerdo con Popper (1973), irrefutables. Otras veces expresan deseos. 
Un caso que contempla la ciencia social es el de la profecía autocumplida o autorrealizadora, (Nagel, 2006) (Merton, 1992) puesto que consiste en afirmar algo erróneo o no probado que resulta verdadero por las acciones que emprenden los hombres al tomar conocimiento de ello. El ejemplo típico fue lo que sucedió cuando se corrió el rumor falso de que un banco iba a quebrar, lo que determinó, en definitiva, su quiebra. En algunas áreas de los estudios sociales se describe este hecho con el denominado "Teorema de Thomas": "Si una situación es definida como real, esa situación tiene efectos reales", lo que también denominaba Claude Levi Strauss como "eficacia simbólica".

Por otra parte Karl Popper (1984) (1985) cuestiona a aquellos que consideran que la tarea de las ciencias sociales es proponer profecías históricas ${ }^{4}$ y que éstas son necesarias, si se desea que el comportamiento social y político siga caminos racionales. A esa doctrina la llama "Historicismo". Para Popper, el historicismo utiliza, en el plano metodológico, un enfoque holístico, dentro del cual se quiere encontrar "leyes históricas" determinísticas. Sin embargo, es imposible, captar la totalidad de los aspectos involucrados en un fenómeno social, pues ello supondría una mente omnisciente, hecho que, científicamente hablando, es una quimera.

\section{Prospectiva}

La "Prospectiva", es la última variedad de anticipación del futuro y puede ser definida como un conjunto de metodologías o técnicas que son usadas con el objetivo de estudiar posibles escenarios futuros y su probabilidad, para tomar decisiones racionales, económicas o políticas.

En el campo de las metodologías prospectivas se llama "Proferencia" al conjunto de técnicas que tienen en común el basarse en el pasado para construir el futuro, incluyendo tanto las proyecciones, las extrapolaciones de tendencias y la redacción de guiones de futurición. Todas estas técnicas determinan un "Futurible", un futuro posible, considerando el estado de cosas actual.

Por otro lado "Prospectiva" es una actitud que "procura hacer más probable que otros, el futuro objetivado como deseable." (Grau, 1999) Por esto este enfoque, a diferencia de la proferencia, parte del futuro (deseable) para llegar al presente, no limitándose a lo meramente posible.

Considerando que la prospectiva se sitúa dentro de una Planificación estratégica, es posible comprender entonces porque se afirma que la Prospectiva "inventa el medio que necesita para cumplir su fin" (Grau, 1999), es decir planifica las acciones necesarias para alcanzar ese futuro deseable o "futurable".

De modo que la Prospectiva sería "la capacidad para percibir, modelizar y eventualmente construir modelos de sociedades futuras mediante la anticipación de nuevas configuraciones - elaboradas de alguna manera - que satisfagan tanto las aspiraciones que motivan a los hombres como las exigencias técnicas imprescindibles para esos logros." (Grau, 1999). Desde un enfoque epistemológico la prospectiva se acerca a lo que Popper (1985) denomina "Ingeniería social gradual"5 en contraposición a las teorías "historicistas". 


\section{Prospectiva y Proyecto}

Por otra parte es posible homologar el concepto de proyecto con un enfoque prospectivo. Recordemos que algunos definen "Proyectar" como el intento por conocer algo con anticipación: prever algo que sucederá.

Mientras que otros consideran a "Proyectar" como el conjunto de acciones que se llevan a cabo en el presente para lograr que las cosas sucedan en el futuro. Este segundo enfoque es más ajustado que el primero que sólo se limita a anticipar hechos. El proyecto hace que los hechos sucedan. (Cravino, 2005)

De este modo es posible concretar algún tipo de "anticipación” del futuro.

Programación Prospectiva: Concretizar los fines en objetivos y programar la acción a realizar. El objetivo fundamental se descompone en una serie de sub-ojetivos que se van graduando en el tiempo, desde los más lejanos a los más cercanos. Cuando ponemos plazos a los objetivos los llamamos metas. (Meta es todo objetivo situado en el tiempo).

Plan: conjunto de decisiones ${ }^{6}$ que produce el avance. (Grau, 2002)

Elaboración de Escenarios ${ }^{7}$ : Conjunto de hipótesis descriptivas acerca del Contexto Futuro en que puede estar una organización.

Los escenarios se pueden clasificar en: esperado o probable, optimista (de máxima o techo) o pesimista (de mínima o piso), a corto plazo- mediano - largo.

En síntesis, el pensamiento de diseño no predice el futuro, sino que tiene una propiedad performativa: Hace que las cosas sean, construye literalmente el futuro.

\section{Características del Pensamiento de Diseño}

\section{Innovador}

Una cuestión que parece obvia pero no lo es, implica que el pensamiento de diseño es heurístico y creativo. Ante contextos inciertos y cambiantes, la fórmula de la tradición fracasa y hace falta innovar. Históricamente innovar parecía significar improvisar, lo cual puede interpretarse como el rechazo a soluciones formularias, estandarizadas y repetidas, y no un mero tanteo a ciegas. No es casual que diversos autores como Pierre Thuillier (1983) al dar cuenta de la primera revolución técnica de fines de la Edad Media recurran al concepto de "ingeniosos" o "inventores" para caracterizar a aquellos que tenían la capacidad de resolver problemas prácticos. O Joel Mokyr (1993) quien define a la innovación en el mundo contemporáneo como la palanca de la riqueza. Podríamos seguir abundando en este concepto pero ya hay mucho escrito al respecto.

Desde la educación David Perkins (1995) define a la creatividad como aquello que:

- Incluye dimensiones estéticas y prácticas;

- Se focaliza en propósitos y resultados;

- Se basa en la movilidad de las ideas;

- Va más allá de las fronteras del pensamiento;

- Se piensa en términos de proyectos y no de problemas aislados;

- Tiene en cuenta diferentes puntos de vista. 
La creatividad es un valor del siglo XX, anterior a ello, lo que valía era la subordinación a la regla y el apego a la tradición. De ahí la importancia que adquiriría la "autoridad", es decir aquello o aquel que define las reglas. Innovar implica entonces también romper una regla. Por eso la innovación está ligada a la noción de vanguardia donde se quiebra una tradición. (Cravino, 2011)

Anthony Giddens (1994) diferencia las sociedades tradicionales de aquellas, actuales, post-tradicionales. En las sociedades tradicionales el pasado tiene una poderosa influencia sobre el presente y las prácticas ya establecidas a su vez son usadas para organizar el tiempo y el espacio en el futuro. Para Giddens (1994) la tradición incluye el ritual y se conecta con lo que él denomina noción formularia de la verdad; tiene guardianes y a diferencia de la costumbre, tiene una fuerza vinculante con un contenido moral y emotivo a la vez. Por el contrario, en la sociedad postradicional la rutina carece de sentido pues no hay garantía que el futuro sea igual que el presente. La duda y la incertidumbre presente en las sociedades postradicionales nos alertan que todo puede estar sujeto a revisión y que ninguna práctica o conocimiento es definitiva. Es por ello que Giddens caracteriza a la modernidad como experimental ya que no existen recetas institucionalizadas.

Para que una sociedad sea creativa sostiene Mokyr (1993) que deben darse una serie de condiciones: "En primer lugar, tiene que existir un conjunto de innovadores ingeniosos y con recursos que estén dispuestos y sean capaces de enfrentarse con su medio físico para mejorarlo (). En segundo lugar, las instituciones económicas y sociales tienen que estimular a los innovadores ofreciéndoles una adecuada estructura de incentivos. () La tercera condición es que la innovación requiere diversidad y tolerancia". Del mismo modo Ilya Prigogine (1997) afirma que es necesario alejarse de los sistemas equilibrados para descubrir situaciones nuevas, o encontrar respuestas a viejos problemas.

La propia noción de "objeto artificial" requiere de la creatividad e imaginación. Un objeto creado por el hombre alguna vez fue "nuevo", y esta novedad implica muchos planos: formales, operativos, funcionales, estéticos, tecnológicos.

Es interesante tomar la definición que brinda Adolfo Corona Martínez (1990) sobre el diseño como un proceso de construcción de conocimiento de aquello que aún no existe: "El diseñador inventa el objeto en el acto mismo de representarlo; esto es, dibuja un objeto inexistente, cada vez con mayor precisión. Esta precisión es un aumento en el detalle, dentro del sistema de reglas de la representación misma. Así el diseño es la descripción progresiva de un objeto que no existe al comenzar la descripción."

No lejos de ello Roberto Fernández (2013, p. 39) señala que el conocimiento proyectual comprende las "transformaciones de la cultura material y del intercambio simbólico entre las sociedades-tanto como un saber sobre las transformaciones a producirse; es decir, un cierto saber-hacer lo que aun no existe".

De manera similar piensa Donald Schon (1998): "Diseñar es una actividad creadora. La conversación reflexiva que un diseñador establece con los materiales de una determinada situación puede generar nuevos descubrimientos, significados e invenciones".

Es por ello que innovar presupone la construcción de conocimiento.

Vale también en este punto diferenciar entre los conceptos de "proyecto fundante" y "proyecto recurrente", categorías establecidas por Fernández (2013, p. 37) quien sostiene que "el proyecto fundante sería aquél que en su concepción y proposición contiene un elemento de 
innovación, una propuesta contributiva a la transformación del problema o necesidad que origina su razón de ser, que pone en marcha la necesidad del proyecto", mientras que define al proyecto recurrente como "una repetición tipológica, a una performance de aplicación serial de una construcción conceptual existente al interior del campo disciplinar/profesional". Para Fernández entonces la primera de estas categorías se corresponde con una lógica de descubrimiento y por ende da origen a la disciplina proyectual como cuerpo de conocimiento, mientras la segunda, por su repitencia, se apoya en el ejercicio profesional.

\section{Holístico}

El pensamiento de diseño es holístico porque no puede ser abordado analíticamente, al menos en su faz creativa. Es holístico en un doble sentido: Porque o bien se diseña o bien no se lo hace: No puede diseñarse "un poquito". Y porque a su vez se debe integrar diferentes dimensiones del objeto o proceso diseñado.

Lo opuesto al pensamiento holístico sería el empleo de un enfoque reduccionista, donde un sistema es reducido a otro con el objeto de simplificar el abordaje de la cuestión. O aquel donde una serie de variables es tomada como constantes pera no afectar la investigación de un único aspecto.

Alberto Sbarra (2015) rescata una frase de Vittorio Gregotti donde les advierte a sus alumnos que profesor decía a sus alumnos: “... ahora ustedes comenzarán a mirar el mundo con ojos de arquitecto", mirada que se impone a todo diseñador como "una visión totalizadora e integradora que incluye el pensamiento proyectual".

Esta integración de múltiples dimensiones, cuya reducción es imposible, aparece en caracterización que realiza Oriol Bohigas (1978):

Un proyecto no es simplemente una exhibición caligráfica ni un objeto expresivo que se agote en sí mismo, a pesar de que el dibujo sea siempre un indicador de contenido y de intenciones no sólo como código directo, sino como transmisor de una poética y hasta de unos métodos. Pero tampoco es la simple ilustración de una teoría arquitectónica, a pesar de que esa teoría debe quedar explicitada en los mismos elementos de representación. Y tampoco es una sucesión indiscriminada de soluciones constructivas, a pesar de que todas ellas están en las condiciones de partida del proyecto y constituyen parte de su base conceptual. Es necesario que la expresión y el contenido coincidan precisamente en la definición de un edificio concreto donde encuentren solución las dificultades de la realidad y la coherencia conceptual, donde dibujo, teoría y tecnología produzcan una arquitectura construíble.

Frijot Capra (2003, p. 28) afirma, respecto a la necesidad de cambio de perspectiva que requiere de un pensamiento sistémico que "El nuevo paradigma podría denominarse una visión holística del mundo, ya que lo ve como un todo integrado más que como una discontinua colección de partes. También podría llamarse una visión ecológica, usando el término «ecológica» en un sentido mucho más amplio y profundo de lo habitual." 
También para un pedagogo como David Perkins (2009) el pensamiento de diseño consiste en una perspectiva integradora que permite tener en cuenta y mantener activas diversas características fundamentales del aprendizaje, considerando, asimismo que constituye una teoría de la acción.

\section{Complejo}

Asimismo el pensamiento proyectual se lo puede incluir dentro del pensamiento complejo, a la manera de Edgar Morin (1998). Recordemos que el pensamiento científico tuvo como una de sus metas, por un lado, la búsqueda de las ideas y las teorías más simples, y por el otro, desde lo metodológico una mirada reduccionista del conocimiento.

Debemos, en principio diferenciar las nociones de "complejo" y "complicado" que muchas veces se emplean como si fuesen sinónimos. Algo es complicado para alguien, Lo complicado es una apreciación cognitiva de un sujeto que caracteriza un hecho por saber como algo difícil o complicado de entender, de tal modo que tal apreciación presenta un rasgo claramente subjetivo. Distinto es el caso de lo complejo, que no depende de una valorización personal de nadie, ya que es una cuestión propia del mismo hecho. Lo complicado puede transformarse en simple o en claro con mayor estudio y dedicación, mientras que lo complejo sigue siéndolo aún después de ser estudiado.

Un sistema complejo es aquel compuesto por partes interrelacionadas, de manera tal que el todo es mayor que la suma de sus partes, y cuya totalidad ofrece propiedades y acciones distintas a las que exhiben sus componentes.

Es por ello que un sistema complejo ofrece una interdependencia entre sus partes y por el otro, la posibilidad de adaptación y de variabilidad de cada una de ellas.

Dada esta complejidad, Edgar Morin ve el mundo como un todo indisociable, y propone un abordaje de manera multidisciplinar y multirreferenciada, donde es necesario entender a los sistemas que conforman el todo como abiertos e inciertos.

\section{Modelístico}

En el pensamiento de diseño es típica la operación a través de modelos o simulaciones. En las tecnologías se usan modelos matemáticos, en el diseño se trabaja por medio de representaciones gráficas o maquetas.

Es frecuente en este tipo de pensamiento la creación de prototipos que sirvan de ensayo y a la vez de propuesta. La noción de prototipo supone además, un cierto control de variables, y la posibilidad de repetición, considerando la resolución dentro del paradigma de la complejidad.

La operatoria a través de modelos permite una economía de recursos y una minimización de los riesgos, típicos de sistemas abiertos e inciertos, pero también permite, desde un enfoque prospectivo, evaluar los distintos escenarios a partir de esta misma operatoria. 


\section{Colaborativo y Coproductivo}

Nigel Cross (2011) definía este punto como el fenómeno de "diseñar juntos", es decir como aquel trabajo que se hace en equipo y cuyo resultado no surge de dividir la tarea en partes sino en trabajar diferentes diseñadores conjuntamente.

La base del trabajo colaborativo es la de compartir el conocimiento y el esfuerzo en función de alcanzar una meta común.

En este tipo de trabajos encontramos diferente modalidades desde la tarea conjunta en torno a un líder o jefe de grupo hasta la coproducción donde es imposible reconocer las huellas particulares de cada diseñador.

El trabajo en equipo, es actualmente valorado en la enseñanza, pues fomenta una inteligencia interpersonal y la socialización de los jóvenes y niños. En este sentido la forma de trabajo en pequeños grupos favorece la construcción de un entorno estimulante donde aprender y compartir, lo cual genera un impacto positivo por el sentido de pertenencia, lo que abre posibilidades a numerosas dimensiones en las que cada estudiante es invitado a aportar, y donde la participación de cada uno se hace necesaria para la concreción de los objetivos planteados.

\section{Multidisciplinario y Transdisciplinario}

Se entiende habitualmente por multidisciplina al esfuerzo conjunto de varias disciplinas diferentes hacia el tratamiento de un determinado objetivo o problema. Generalmente incluye el trabajo colaborativo de personas formadas en diferentes áreas aplicando sus propios saberes y herramientas.

Asimismo, la interdisciplina es definida como el esfuerzo indagatorio convergente entre varias disciplinas que tiene como objetivo el obtener conocimiento acerca de un objeto de estudio nuevo.

De un mismo modo, la transdisciplina se puede concebir como un proceso de construcción del conocimiento a través de la creación de un nuevo marco conceptual que se hace necesario para hacer posible el abordaje de un nuevo objeto en común. La transdisciplina está relacionada con el cruce de fronteras disciplinares en la construcción del conocimiento. (Cravino, 2014)

Por otra parte el crecimiento exponencial del saber aún, en una misma área de conocimiento, fragmenta las disciplinas y las especializa.

El pensamiento complejo apunta a que es necesario articular e integrar las distintas dimensiones de lo real, reconociendo por un lado los quiebres y compartimientos del saber, en un contexto en el que la realidad exige enfoques multi, inter y transdisciplinares. Para lo cual hay que establecer una estrategia reflexiva que tiene una intención globalizadora o abarcativa de los fenómenos, que a su vez, reconoce la especificidad de las partes En nuestra caracterización, la noción de multidisciplina viene tanto del enfoque holístico y complejo como del trabajo colaborativo o coproductivo. El diseño es por definición una actividad integral que implica múltiples dimensiones. De tal manera que coincidimos con Sarquis y Buganza (2009) quienes sostienen que "La complejidad se nutre de la explosión disciplinaria fomentándola, al mismo tiempo que determina la multiplicación de las disciplinas, exigiendo una coordinación más cercana entre ellas. () Sin embargo, multidisciplina- 
riedad e interdisciplinariedad quedan inscriptas en el marco de la investigación disciplinaria. He ahí el gran reto de la transdisciplina: recomponer a partir de la fragmentación".

Rolando García (2006, p. 33) nos aporta un poco más de claridad cuando desde una epistemología de raíz piagetiana elude la simplificación y sostiene que "Los sistemas complejos están constituidos por elementos heterogéneos en interacción -y de allí su denominación de complejos-, lo cual significa que sus subsistemas pertenecen a los «dominios materiales» de muy diversas disciplina" que es necesario articular considerando los distintos enfoques disciplinares existentes, asumiendo además sus difusos límites.

\section{Mirada Micro y Macro}

La cuestión de la escala en el diseño implica necesariamente ir del todo a las partes y de las partes al todo, puesto que el diseño posee un enfoque holístico e implica una habilidad integradora.

El diseño es integral porque necesita de una coherencia interna que le impone el diseñador. Y en esa búsqueda de coherencia es necesario ir del detalle a las totalidades. En este sentido dice Donald Schon (1998, p. 50) que un diseñador es "alguien que transforma situaciones indeterminadas en determinadas ( ) Los diseñadores construyen e imponen una coherencia propia. () El análisis y la crítica juegan un papel relevante dentro de un proceso más amplio."

"Dios está en los detalles", frase atribuida a Gustave Flaubert y tomada por Ludwig Mies van der Rohe, quien la transformó en uno de sus lema, plantea esta fascinación por lo micro.

Por otro lado, la noción de coherencia surge del equilibrio entre el enfoque micro y el macro que podemos resumir en la sentencia clásica "de no dejar que el árbol tape al bosque" o en aquella otra que se refiere a la "unidad en la diversidad".

\section{Reflexión en la acción}

En primer lugar, para caracterizar este aspecto, debemos recurrir a la investigación-acción, típica de los contextos educativos. La investigación acción es la que se realiza sobre la propia práctica. Y este modelo de investigación, surgió, en primer lugar, en el ámbito educativo, en el interior de las escuelas.

Como asevera Kemmis (1988) "la acción práctica, y el modo de pensamiento asociado a ella, consiste en hacer la acción. Es esencialmente arriesgada: está guiada por ideas morales, general y, a veces, trágicamente conflictivas, relacionadas con el bien de la humanidad: implica sopesar circunstancias y hacer juicios de manera que se pueda actuar correctamente en situaciones humanas y sociales concretas". El razonamiento práctico, a diferencia del técnico, siempre incluye juicios morales y de ahí el peso de las decisiones.

La idea de acción práctica está ligada a la caracterización del diseño como una actividad útil (en sentido que un objeto de diseño no es una pieza artística, por más que posea valores estéticos) y productiva (a diferencia de las ciencias que son explicativas y las humanidades que son reflexivas). (Archer, 12992) Estas dos características sitúan al diseño en el mundo de la acción, dependiendo de las decisiones que realizan los seres humanos. 
Asimismo estas decisiones dependen de un conjunto de intenciones y valores que poseen los sujetos que actúan en un complejo mundo (social y natural). Recordemos que para Giddens (1997) "Desde un punto de vista analítico, sería más correcto decir que todas las áreas de la actividad social están gobernadas por decisiones -tomadas frecuentemente, aunque no siempre, sobre la base de alguno u otro tipo de conocimiento experto-. Quién toma esas decisiones y cómo lo hace es fundamentalmente un problema de poder. Una decisión es siempre la elección de alguien y en general todas las elecciones, incluso las más aparentemente insignificantes, tienen como telón de fondo un conjunto de relaciones de poder preexistentes".

Enfocándose en el funcionamiento de las organizaciones Chris Argyris (1999, p. 322) concibe la llamada Teoría de acción, para estudiar cómo los seres humanos diseñan sus acciones en situaciones complejas e inciertas. Argyris concibe al individuo como un ser que construye un tipo de conocimiento (práctico, tácito) para orientar sus acciones, las que ejecuta y evalúa para que, a partir de las consecuencias de dichas acciones se puedan estudiar los cambios resultantes.

Esta teoría deberá cumplir con tres condiciones:

- Debe ser utilizada para describir y comprender la realidad (Es decir, definir problemas);

- Debe inventar nuevas soluciones a dichos problemas;

- Y debe fijar el curso de la acción, qué decisiones de deben tomar y cómo evaluar los resultados.

Para definir esta característica aplicada al pensamiento proyectual recurriremos a algunas citas de Donald Schon ${ }^{8}$ :

Un proceso de diseño competente es una forma de conocimiento en la acción.

( ) Esto ayuda a explicar por qué los estudiantes deben practicar si quieren aprender a diseñar -y sugiere, además, que su práctica debe implicar la reflexión en la acción- pero no explica por qué no pueden aprender a diseñar en el orden propio de un currículum profesional normativo: primero teorías en el aula, después un prácticum que las aplica. (Schön, 1998, p. 93)

La descripción del propio conocimiento en la acción que uno posee, es en sí misma, una competencia, y los diseñadores pueden poseerla en mayor o menor medida. Los diseñadores pueden aprender a mejorar las descripciones del diseño -a-hacerlas más complejas, precisas y útiles para la acción- por medio de una reflexión continuada sobre sus propias ejecuciones competentes. (Schön, 1998, p. 95)

Los profesionales del diseño ( ) tratan con la incertidumbre, la singularidad y el conflicto. Las situaciones no rutinarias de la práctica resultan, al menos en parte, indeterminadas y se les debe proporcionar algún tipo de coherencia. Los prácticos competentes aprenden a experimentar sobre el marco conceptual, lo que les sirve para imponer una determinada coherencia sobre situaciones confusas y, a partir de ahí, descubrir consecuencias e implicaciones de los marcos que han elegido. De vez en cuando, sus esfuerzos para poner orden en 
una determinada situación provocan resultados imprevistos ( ) Este conjunto de cuestiones - la reformulación del problema, el experimento riguroso, el descubrimiento de consecuencias e implicaciones, la charla retrospectiva y las respuestas a este tipo de charla - es el que configura una conversación reflexiva con los materiales de una situación: el arte de la práctica profesional que caracteriza el diseño" (Schön, 1998, p. 146)

No obstante, Schon sostiene que el conocimiento así obtenido no puede ser verbalizable? cuestión que hemos discutido en otro escrito y que rechazamos. (Cravino, 2018)

\section{Aprender de los demás. Estudios de casos y casos problemas}

En el pensamiento de diseño es necesario generar empatía con el futuro usuario, aprender a escuchar sus deseos, necesidades e inquietudes. Asimismo se debe indagar críticamente sobre alternativas de soluciones que se han empleado para resolver el problema formulado y poner en crisis tanto las soluciones como la definición del propio problema.

El estudio de casos o casos problema permite tanto estudiar soluciones pasadas como ensayar nuevas alternativas. Es una estrategia típica de simulación profesional donde los aprendices "juegan" con variables y situaciones semejantes a las que se enfrentan los profesionales.

En síntesis:

Desde un punto de vista pedagógico se sabe que el aprendizaje complejo implica la integración de conocimientos, la coordinación de ciertas habilidades que son cualitativamente diferentes, además de la transferencia de lo aprendido en un entorno educativo al ámbito de la vida y el trabajo diarios. El interés actual en el aprendizaje complejo se manifiesta en algunos enfoques como el estudio de casos y los casos problema. Se habla entonces de resolución colaborativa de problemas (Nelson, 2000), constructivismo y ambientes constructivistas de aprendizaje (Jonassen, 2000), aprender haciendo (Schank, 2000) (Dewey, 1967) (Dewey, 1998). Aunque todas estas propuestas tienen elementos distintos manifiestan en común el hecho que desarrollan "tareas auténticas de aprendizaje" basadas en fenómenos o episodios de la vida diaria como impulso para la enseñanza.

La idea principal que sustentan estos enfoques es que la realización de las mencionadas tareas ayudan a los aprendices a integrar conocimientos, habilidades y actitudes, los estimulan para que aprendan a coordinar habilidades constitutivas, y facilitan la transferencia de lo aprendido a nuevas situaciones problemáticas. Estas cuestiones son típicas del pensamiento de diseño, que Nigel Cross y Bruce Archer pretendían fomentar en el sistema educativo. Por ende, después de indagar en estos "nuevos" modelos de aprendizaje, los que actuamos en carreras proyectuales nos encontramos como aquel personaje de Moliere que "hablaba en prosa sin saberlo", ya que se afirma que "El aprendizaje mediante ejemplos entronca con contextos reales; los alumnos tienen que arreglárselas ante situaciones complejas y pensar como profesionale" (Jonassen, 2000, p. 228). Puesto que se plantea como "innovación" pedagógica, lo que los profesores de arquitectura y diseño, hacemos desde siempre... Es decir, utilizando un "pensamiento de diseño"!!! 


\section{A modo de conclusión}

Como ya hemos dicho la expresión "pensamiento de diseño" se utiliza hoy en día en un sentido más amplio, como un enfoque para resolver problemas fuera de la práctica de diseño profesional, como en los negocios y diferentes contextos sociales donde la incertidumbre y la ambigüedad son moneda corriente.

De este modo, si el pensamiento proyectual o pensamiento de diseño se refiere a las estrategias creativas que los diseñadores utilizan durante el proceso de diseño, estas estrategias pueden ser replicadas en diferentes ámbitos, permitiendo la reconceptualización de situaciones complejas y su posterior solución.

\section{Notas}

1. Leyes: Afirmaciones universales condicionales, confirmadas o corroboradas empíricamente que aseguran la ocurrencia del consecuente dada la ocurrencia del antecedente. No hacen referencia a situaciones espacio temporales determinadas y tienen pretensión de validez universal.

2. Vale señalar que, desde un punto de vista científico, es imposible verificar las leyes generales, siendo, entonces hipótesis corroboradas.

3. Afirmaciones que describen el comportamiento de una o más variables en el curso del tiempo. Son singulares históricas (se refieren a eventos situados), estimativas (se refieren al comportamiento promedio de las variables) y volátiles (persisten mucho tiempo o cambian bruscamente).

4. Por ejemplo hablar de destino, misión histórica, etc. Nota: Gregorio Klimovsky (1998) sintetiza la posición historicista: "1) Historicista es una denominación inventada por Popper para aludir a este tipo de intelectual o estudioso que centra la clave de su concepción en la formulación de leyes de tendencia o de proceso, no universales; 2) sus anticipaciones sobre el futuro tienen el carácter de profecía; 3) sus afirmaciones tienen carácter fatalista, porque hágase lo que se haga, como la tendencia está dada, el fin es concebido como inevitable y 4) el proceso o la tendencia puede acelerarse o retardarse, pero no puede corregirse el resultado"

5. "Por otro lado la ingeniería social gradual se sustenta en el desarrollo progresivo "Este no debería ir e una sola dirección, porque en este caso no podría preverse, sino que debería llevarse a cabo mediante ajustes, acomodaciones y adaptaciones". (Klimovsky-Hidalgo, 1998) 6. "En cada nivel de decisión hay siempre una posible elección de estrategias que verifica la misma secuencia: 1) objetivos; 2) recursos, 3) limitaciones; 4) estrategias; 5) cursos de acción y 6) evaluación de resultados y de estrategias" (Grau, 2002)

7. Escenario: Es el espacio definido para la acción estratégica. Es más una representación mental de la realidad en un momento dado que una realidad en sí mismo. Puede ser también construido a través de la acción estratégica.

8. Ver también: (Cravino, 2009, p. 65-69) (Cravino, 2018, p. 163-185)

9. "Igual que el conocimiento en la acción, la reflexión en la acción es un proceso que podemos llevar adelante sin ser capaces de decir lo que estamos haciendo...." (Schon, 1998, p. 40) 


\section{Listas de Referencias Bibliográficas}

Archer, Bruce; Baynes, Ken; Roberts, Phil (1992) The nature of research into Design and Technology education", Loughborough University Dept. of Design \& Technology, Leicestershire. En: https://dspace.lboro.ac.uk/dspace-jspui/bitstream/2134/1687/3/Archer_Baynes_Roberts.pdf

Archer, L. Bruce (1965) Systematic Method for Designers, Council for Industrial Design, London: 1965, Based on a series of articles in Design magazine (1963-64).

Archer, L. Bruce (1979) "Design as a discipline" Design Studies Vol1, No 1, Julio 1979 En: https://monoskop.org/images/2/2f/Archer_Bruce_1979_Design_as_a_Discipline.pdf

Argyris, Chris (1999) Conocimientos para la acción. Granica, Buenos Aires

Bohigas, Oriol (1978) Proceso y erótica del diseño. Editorial La Gaya Ciencia, Barcelona

Bunge, Mario (1989) Seudociencia e Ideología, Alianza Universidad, Madrid.

Capra, Fritjof (2003) La Trama de la vida, Editorial Anagrama, Barcelona

Corona Martínez, Alfonso (1990) Ensayo sobre el Proyecto, Editorial CP67, Buenos Aires

Cravino, Ana (2014) "Disciplina, integración y transferencia en la construcción del saber proyectual" en rda - revista de la facultad de arquitectura de la UCSF, Universidad Católica de Santa Fe, año 2, № 2, septiembre de 2014.

Cravino, Ana (2001) “Creatividad y paradigmas" en Reflexión Académica en Diseño y Comunicación Año XII, Vol. 16, Agosto 2011, Buenos Aires

Cravino, Ana (2005) Reflexiones sobre la Teoría y la Crítica, Editorial Praia, Buenos Aires

Cravino, Ana (2009) "Releyendo a Donald Schön" en Reflexión Académica en Diseño y Comunicación 2009. Año X, Vol. 11, Buenos Aires, Febrero 2009, Pág. 65-69.

Cravino, Ana (2018) Enseñar Diseño: La emergencia de la teoría en Cuadernos del Centro de Estudios en Diseño y Comunicación. Ensayos No.67, Buenos Aires, mayo 2018, Pág.163-185.

Cross, Nigel (2001) "Designerly Ways of Knowing: Design Discipline Versus Design Science" En Design Issues: Vol 17, No 3, Summer 2001, MIT. En http://users.metu.edu.tr/baykan/ arch467/Readings/Cross01.pdf

Cross, Nigel (2011) Design Thinking, Berg, Nueva York. En: https://designpracticesandparadigms.files.wordpress.com/2013/01/wk1_cross_designthinking.pdf

Dewey, John (1967). Experiencia y Educación. Losada, Buenos Aires

Dewey, John (1998) Democracia y Educación, Morata, Madrid

Fernández, Roberto (2013) Inteligencia proyectual Un manual de investigación en arquitectura Colección UAI - Investigación, Editorial Teseo, Buenos Aires

Gaeta, Rodolfo (1996) Modelos de explicación científica: problemas epistemológicos de las ciencias naturales y sociales, Eudeba, Buenos Aires

Garcia, Rolando (2006) Sistemas complejos. Conceptos, método y fundamentación epistemológica de la investigación interdisciplinaria, Gedisa, Barcelona

Giddens, Anthony (1994) Consecuencias de la modernidad, Alianza, Madrid.

Giddens, Anthony (1997) "La vida en una Sociedad Post-Tradicional" en Revista Agora $N^{o}$ 6, Buenos Aires, Verano de 1997

Grau, Jorge (2002) Tecnologia e Historia, Fundec, Buenos Aires. 
Grau, Jorge E.(1999) Tecnología y Prospectiva, Fundec, Buenos Aires

Hempel, Carl (1979) "La función de las leyes generales en la historia" y "Aspectos de una explicación científica”, en Hempel, Carl (1979) La lógica de la Explicación, Paidós, Buenos Aires.

Jonassen, David (2000), Capítulo 10 "El Diseño de Entornos Constructivistas de Aprendizaje", en Reigeluth, Charles (2000) Diseño de la Instrucción: Teorías y modelos. Un nuevo paradigma de la teoría de la instrucción. Aula XXI Santillana, Madrid, Pág. 225-250

Kemmis, Stephen (1988) El curriculum: más allá de la teoría de la reproducción. Morata, Madrid.

Klimovsky, Rodolfo - Hidalgo, Cecilia (1998) La inexplicable sociedad .Cuestiones de epistemología de las Ciencias Sociales. AZ Editores. Buenos Aires

Marques, Gustavo (1999) "Predicción en economía" en SCARANO, Eduardo (1999) Metodología de las ciencias Sociales. Lógica, lenguaje y racionalidad, Ediciones Macchi, Buenos Aires.

Merton, Robert K (1992) Teoría social y estructura social, FCE, México,

Mokyr, Joel (1993) La Palanca de la Riqueza. Creatividad tecnológica y progreso económico. Alianza, Madrid

Morin, Edgar (1998) Introducción al pensamiento complejo, Gedisa, Barcelona.

Nagel, Ernest (2006) La estructura de la ciencia, Paidós, Barcelona

Nelson, Laurie Miller (2000) Capítulo 11 "Resolución de Problemas en colaboración" en Reigeluth, Charles (2000) Diseño de la Instrucción: Teorías y modelos. Un nuevo paradigma de la teoría de la instrucción. Aula XXI Santillana, Madrid. p. 251-278

Perkins, D. (1995). La escuela inteligente. Del adiestramiento de la memoria a la educación de la mente. Gedisa, Barcelona

Perkins, David (2009). El aprendizaje pleno. Paidós. Buenos Aires.

Popper, Karl (1973) La lógica de la investigación científica, Editorial Tecnos, Madrid

Popper, Karl (1984) La miseria del historicismo, Alianza, Madrid

Popper, Karl (1985) La sociedad abierta y sus enemigos, Hyspamérica, Bs.As.,

Popper, Karl (1989) "Predicción y profecía en las ciencias sociales", en Conjeturas y refutaciones. El desarrollo del conocimiento científico, Paidós, Barcelona,

Prigogine Ilya (1997) “El desorden creador" en Revista Iniciativa Socialista No 46 (primera época de la actual revista Trasversales), otoño 1997

Prigoyine, Ilya (1996) El fin de las Certidumbres, Andrés Bello, Santiago de Chile.

Rowe, Peter (1987) Design Thinking, MIT Press, Cambridge. http://www.egyptarch.gov.eg/ sites/default/files/pdf/Books/Design\%20\%20Thinkng.pdf

Salmon, Merrilee H. (2002) "La explicación causal en las Ciencias Sociales" en González, Wenceslao (ed.) (2002.) Diversidad de la explicación científica. Ariel, Barcelona

Sarquís, Jorge; BUGANZA, Jacob (2009) "La teoría del conocimiento transdisciplinar a partir del Manifiesto de Basarab Nicolescu” en Fundamentos en Humanidades No 1, Vol. 19, 2009, Universidad Nacional de San Luis, Pág. 43-55

Sbarra, Alberto -Morano, Horacio - Cueto Rúa, Verónica (2015) “El Mundo como Proyecto" en XXXIV Encuentro Arquisur - XIX Congreso "Ciudades Vulnerables. Proyecto o Incertidumbre" Universidad Nacional De La Plata 
Schank, Roger- Berman, Tamara- Macpherson, Kimberli (2000). Capítulo 8 "Aprender a través de la práctica” en, Reigeluth, Charles (2000) Diseño de la Instrucción: Teorías y modelos. Un nuevo paradigma de la teoría de la instrucción. Aula XXI Santillana, Madrid Pág. 173-192.

Schon, Donald (1998) La formación de Profesionales reflexivos, Paidós, Madrid.

Schuster, Félix Gustavo (2005) Explicación y Predicción. La validez del conocimiento en ciencias sociales, CLACSO, Buenos Aires.

Simon, Herbert (1996) The science of the artificial, MIT, Cambridge, Mas

Thuillier, Pierre (1983) La trastienda del sabio. Editorial Hachette, Barcelona

Abstract: The objective of this paper is to reflect on the characteristics of design thinking, typical of Design disciplines. This type of thinking, to break with other modalities and cognitive traditions, is presented today as an opportunity for various disciplines.

Keywords: Design thinking - Projective thinking - design research - prospective

Resumo: O objetivo deste artigo é refletir sobre as características do design thinking, típicas das disciplinas de desenho. Esse tipo de pensamento, para romper com outras modalidades e tradições cognitivas, é apresentado hoje como uma oportunidade para várias disciplinas.

Palavras chave: Design thinking - Pensamento projetual - pesquisa de design - prospectiva [Las traducciones de los abstracts fueron supervisadas por el autor de cada artículo] 